Voix et Images

volxetimages

\title{
Bibliographie sélective de Jacques Ferron
}

\section{Pierre Cantin}

Volume 8, numéro 3, printemps 1983

Jacques Ferron

URI : https://id.erudit.org/iderudit/200402ar

DOI : https://doi.org/10.7202/200402ar

Aller au sommaire du numéro

\section{Éditeur(s)}

Université du Québec à Montréal

\section{ISSN}

0318-9201 (imprimé)

1705-933X (numérique)

Découvrir la revue

\section{Citer ce document}

Cantin, P. (1983). Bibliographie sélective de Jacques Ferron. Voix et Images, 8(3), 465-473. https://doi.org/10.7202/200402ar d'utilisation que vous pouvez consulter en ligne.

https://apropos.erudit.org/fr/usagers/politique-dutilisation/ 


\title{
Bibliographie sélective de Jacques Ferron
}

\author{
par Pierre Cantin, Collage de l'Outaouais
}

Des premiers écrits publiés dans des journaux étudiants /le Brébeuf et /e CarabinLaval), durant les années 1930 et 1940, jusqu'aux plus récentes collaborations au Courrier médical et aux recensions dans Livre d'ici, Jacques Ferron a fait paraitre près de 1,200 textes dans la presse périodique du Québec et du Canada anglais. Une quarantaine d'oeuvres publiées ou. rééditées s'ajoutent à cette impressionnante production. En 1976, Diane Potvin en a dressé un premier inventaire' que nous avons complété et corrigé en $1980^{2}$, dans le cadre d'une recherche systématique entreprise pour une thèse de doctorat soutenue en juin $1981^{3}$. Pour ne pas faire double emploi, il était donc superflu d'inclure la production entière du conteur et de l'essayiste dans un numéro consacré à l'auteur de l'Amélanchier, comme il est également impensable de vouloir donner ici la liste complète de plus d'un millier de critiques et de documents divers parus sur l'homme et l'oeuvre.

Des écrits de ferron, la présente bibliographie ne retient que les oeuvres en librairie ainsi que les rares pièces radiophoniques demeurées inedites. Les livres et les radiothéátres sont classées respectivement par ordre de parution et de diffusion. L'inventaire de la critique, quant à lui, regroupe, par ordre alphabétique d'auteurs, un choix d'essais et un choix des études les plus importantes parues dans des livres et certains périodiques. Une liste des thèses et des mémoires complète la sólection.

\section{A. Oeuvres parues en librairie}

1. L'Ogre, (pièce en quatre actes), (Montréal), les Cahiers de la File indienne, (1949), $83 \mathrm{p}$.

Création à la scène (remaniée par Ferron): le 17 novembre 1958, au studio d'Essai du Théâtre-Club, à Montréal.

Mise en scène: Marcel Sabourin.

2. La Barbe de François Hertel, (sotie), suivie de: le Licou, (pièce en un acte), (Montréal). Editions d'Orphée, (1951), $40 \mathrm{p}$.

Le Licou: création à la scène: le 24 juin 1958, au Studio d'Essai du Théâtre-Club, à Montséal, par la Troupe de l'Errant canadien.

Mise en scène: Marcel Sabourin. 
Reprise: à Paris, à la Maison du Canada.

Mise en scène: Marcel Sabourin.

3. Le Dodu ou le Prix du bonheur, (pièce en un acte), (Montréal), Ėditions d'Orphée, (1956), 91(1) p.

Création à la scène: le 24 juin 1958, au Studio d'Essai du Theastre-Club, à Montréal, par la Troupe de l'Errant canadien.

Mise en scène: Marcel Sabourin.

Reprise: en mai 1963, au Théatre-Club, par la Troupe.

Mise en scène: Roger Tótreault.

en janvier 1964, par le Théâtre de l'École.

Mise en scène: Guy L'Écuyer.

4. La Barbe de François Hertel; Le Licou, (Montréal), Éditions d'Orphée, (1956), 110 p.

Réédition des deux textes publiés en 1951.

5. Tante Élise ou le Prix de l'amour, (pièce en un actel, (Montréal), Éditions d'Orphée, (1956), 102(1) p.

6. Le Cheval de Don Juan, (pièce en trois actes), (Montréal), Éditions d'Orphée, (1957), 223(1) p.

Création à la scène: en septembre 1958, par la troupe Les Audacieux, à Longueuil; jouée à nouveau, par la mẻme troupe, à Rimouski, en janvier 1961 et à Lévis, en mars 1961.

Reprises: par un groupe d'étudiants, à la Polyvalente Louis-Joseph-Papineau, à Papineauville, en 1974;

par le Théâtre du Petit Brûlé (?), à Laval-Ouest, durant l'été 1979.

7. Le Licou, Montréal, Éditions d’Orphée, (1958), $103(1)$ p.

Troisième édition de la pièce publiée en 1951.

8. Les Grands Soleils, (pièce en trois actes), (Montréal), Éditions d'Orphée, (1958). $180(1)$ p.

Création à la scène (dans une version remaniée): le 27 janvier 1968, par le Théătre du Nouveau Monde et présentée ensuite en tournée dans 44 villes du Québec, de l'Ontario et du Nouveau-Brunswick. Jouée au Théattre Port-Royal de la Place des Arts, à Montréal, du 25 avril au 26 mai 1968.

Mise en scène: Albert Millaire.

Reprise (dans une version abrégée): à l'été de 1977, au Théâtre du Bois de Coulonge, à Québec.

Mise en scène: Jean-Marie Lemieux.

9. Contes du pays incertain, (Montréal), Éditions d'Orphée, (1962), 200 p.

Recueil de dix-sept contes, dont seize ont paru, entre 1953 et 1960, dans I'Information médicale et paramédicale, Amérique française et Situations.

Traduction: dix contes, par Betty Bednarski. Inclus dans le recueil paru sous le titre Tales from the Uncertain Country, Toronto, Anansi, 1972, v, 101 p.

10. Cornoir. (roman), (Montréal), Editions d'Orphé, (1962), 99 p.

Traduction: sous le titre Dr. Cotnoir, par Pierre Cloutier, Montréal, Harvest House, (1973). 86 p. (coll. "French Writers of Canada). 
11. La Tête du roi, (pièce en quatre actes), (Montréal, Association générale des étudiants de l'Université de Montréal, 1963), 93 p. (coll. "Cahiers", 10).

12. Cazou ou le Prix de la virginité, (pièce en un acte), (Montréal), Éditions d'Orphée, (1963), 8611) p.

Création à la scène: au Centre des Loisirs de l'Immaculée-Conception (?), à Montréal. Reprise: au Café Le Hibou, à Ottawa, en mai 1973.

Mise en scène: Denis Blais.

13. Contes anglais et autres, (Montréal), Ėditions d'Orphée, (1964), 153 p.

Recueil de vingt-trois contes parus dans I'Information médicale et paramédicale et dans Amérique trançaise entre 1952 et 1964.

Traduction: sous le titre "English Tales and Other Stories", par Larry Shouldice (thèse, M.A., Université de Sherbrooke, 1971). Huit contes ont été également tra duits par Betty Bednarski et intégrés dans le recueil Tales from the Uncertain Country (voir entrée 9, supra).

14. "La sortie", (pièce en un acte), Montréal, Écrits du Canada frạ̧̧ajeq 19 (1965), p. (109)-147.

La page de titre précise: "supplément à Tante Elize (sic)".

Création a la scène: le 14 septembre 1965; par le Théâtre de la Place, à la Place Ville-Marie, à Montréal, avec le Triangle et le Hamac d'André Rlchard et /e Train de Michel Tremblay.

Mise en scéne: Pascal Desgranges.

15. La Nuit, (roman), (Montréal), Editions Parti pris, (1965), 134 p. (coll. "Paroles", 4).

16. Papa Boss, (roman), (Montréal), Ėditions Parti pris, (1966), 142 p. (coll. "Paroles", 8).

17. Contes, édition intégrale: Contes anglais; Contes du pays incertain; Contes inédits, Montreal, Éditions HMH, 1968, 210 p. (coll. "l'Arbre", G-4).

Le recueil reprend, avec de très rares variantes, les deux ouvrages de contes publiés en 1964 et en 1962 ainsi que quatre contes parus dans /'Information médicale et paramédicale, Amérique française et Chátelaine, entre 1955 et 1965.

Note: plusieurs de ces contes, traduits, ont été repris dans des anthologies et des revues canadiennes-anglaises. D'autres, traduits en tcheque, en polonais, en russe ou en hébreu, ont paru dans des revues étrangères à l'occasion de numéros spéciaux consacrés à la littérature d'ici.

18. La Charrette, roman, Montréal, Éditions HMH, 1968207 p. (coll. "I'Arbre", 14). Traduction: sous le titre The Cart, par Ray Ellenwood, Toronto, Exile Editions, (1981), 144 p. Piusieurs chapitres avaient précédemment paru dans la revue Exile, en 1977 et en 1981.

19. Théâtre 1: les Grands Soleils; Tante Elise; le Don Juan chrétien, Montréal, Librairie Déom, (1968), 229 p. (Postface) d'André Major.

Reédition de Tante Elise, dans sa forme originelle, des Grands Soleils et du Cheval de Don Juan, versions remaniées. 
20. "Le coeur d'une mère", (pièce en un acte), Montréal, Écrits du Canada français, 25 (1969), p. (55)-94.

21. Historiettes, Montréal, Editions du Jour (1969), 182 p. (coll. "les Romanciers du Jour", R-43).

Reprise de trente-cinq textes (dont certains ont été remaniés) parus dans cinq périodiques V'Information médicale et paramédicale, Liberte, I'Action nationale, Situations et Parti pris), de 1957 a 1969.

22. Le Ciel de Québec, roman, Montréal, Editions du Jour, (1969), 404(1) p. (coll. "les Romanciers du Jour", R-51).

Une partie substantielle de l'ouvrage est composée d'historiettes publiées dans I'Information médicale et paramédicale, de 1968 à 1970.

Un épisodé de l'oeuvre sera adapté pour la scène par Victor-Lévy Beaulieu, sous le titre la Tete de monsieur Ferron ou les Chians, et joube au Théatre d'Aujourd'hui, à Montréal, du ler mars au 8 avril 1979, dans une mise en scène d'André Brassard.

23. L'Amélanchier, récit, Montréal, Éditions du Jour, (1970), 163 p. (coll. "les Romanciers du Jour", R-56).

Traduction: sous le titre The Juneberry Tree, par Raymond Y. Chamberlain, (Montréal, Harvest House, 1975), 157 p. (coll. "French Writers of Canada").

L'oeuvre a été adaptée à la scène par Johane Mongeon et jouée par le Module de Théâtre de l'Université du Québec à Montréal, en novembre 1974.

24. Cotnoir, roman, suivi de: la Barbe de Francois Hertel, Montréal, Éditions du Jour. (1970), 127 p. (coll. "les Romanciers du' Jour", R-57).

Rééditions des textes parus en 1962 et en 1951.

25. Le Salut de I'trlande, roman, Montréal, Editions du Jour, (1970), 221 (1) p. (coll. "les Romanciers du Jour", R-69).

L'oeuvre reprend, avec de nombreuses variantes, une vingtaine de textes parus dans /'Information médicale et paramédicale, en 1966 et en 1967.

26. Les Roses sauvages, petit roman suivi d" "Une lettre d'amour" soigneusement pré sentée, Montréal, Éditions du Jour, (1971), 177 p. (coll. "les Romanciers du Jour", R-75).

Le roman s'inspire largement d'une série de textes parus dans /'Information médicale et paramédicale, sous le titre "Le contentieux de l'Acadie", en 1966 et en 1967. Traduction: sous le titre Wild Roses, a story followed by a "Love letter", par Betty Bednarski, (Toronto), McLelland and Stewart, (1976), 123 p.

27. La Chaise du maréchal ferrant, roman, Montréal, Éditions du Jour (1972), 223(1) p. (coll. "les Romanciers du Jour", R-80).

La première partie du roman (p. 9-60) a paru dans Châte/aine, sous l'appellation de "nouvelle", en avril 1972.

28. Le Saint-Elias, roman, Montréal, Éditions du Jour, (1972), 186 p. (coll. "les Romanciers du Jour", R-85). 
Traduction: sous le titre The Saint Elias, par Pierre Cloutier, (Montreal, Harvest House, 1975), 145 p. (coll. "French Writers of Canada").

29. Les Confitures de coings et autres textes, (Montréal), Editions Parti pris, (1972), 326 p. (coll. "Paroles", 21).

L'ouvrage comprend: Papa Boss, Version corrigée et refondue du texte paru en 1966; les Confitures de coings, version entièrement nouvelle de la Nuit, publiée en 1965 ainsi que deux textes inédịts, la Créance et Appendice aux Confitures de coings ou le Congédiement de Frank Archibald Campbell.

Traduction, sous le titre général Quince Jam, par Ray Ellenwood, (Toronto), Coach House, (1977), 262 p. (coll. "Québec Translation").

Papa Boss et Quince Jam avaient été publiés intégralement dans la revue Exile en 1972 et en 1974.

30. L'Amelanchier, récit, Paris, Éditions Robert Laffont, (1973), 162(1) p.

Réédition de l'oeuvre parue en 1970.

31. Du fond de mon arrière-cuisine, (recueil d'essais), Montréal, Éditions du Jour, (1973), 290 p. (coll. "les Romanciers du Jour:", R-105).

A l'exception de trois textes, les essais réunis ici ont tous paru dans la presse périodique (I'Information médicale et paramédicale, Incidences, Parti pris), de 1962 à 1973. Certains ont été remaniés. Des titres ont été changés.

Traduction: "Claude Gauvreau" et "Les Salicaires", traduits par Ray Ellenwood et publiés dans la revue Exile, en 1976.

32. Théstre 2: le Dodu ou le Prix du bonheur; la Mort de monsieur Borduas; le Permis de dramaturge; la Téte du roi; I'Impromptu des deux chiens, Montréal, Librairie Déom, (1975), 192 p. (Postface) d'André Major.

Le Dodu ou le Prix du bonheur et la Tete du roi sont des reprises intégrales des éditions de 1956 et de 1963. La Mort de monsieur Borduas a d'abord paru dans les Herbes rouges, en 1968, le Permis de dramaturge, dans la Barre du Jour, en 1965 et /Impromptu des deux chiens, en partie, dans Thestre-Quebec, en 1969.

33. Escarmouches. La longue passe, 2 tomes, (Montrél), Leméac, (1975), 391 et $227 \mathrm{p}$. (coll. "Indépendances", 3). "Le texte épars (préface)" par Jean-Marcel (Paquette).

Recueil de 178 textes (historiettes, chroniques littéraires, lettres aux journaux, prises de position, etc.) parus dans une dizaine de revues et de journaux québécois, de 1954 à 1974. Une lettre inédite de l'écrivain Pierre Baillargeon s'ajoute à ces écrits généralement rédigés sur le ton de la polémique.

34. Les Confitures de coings et autres textes, textes suivis de: le Journal des Confitures de coings, (Montréal), Éditions Parti pris, (1977), 293 p. (coll. "Projections libérantes", 3).

Réédition des quatre textes publiés en 1965.

35. L'Amélanchier, récit, (Montréal-Nord), VLB éditeur, (1977), $149 \mathrm{p}$.

Réédition de l'oeuvre parue en 1970. 
36. "La Nuit" de Jacques Ferron, présentée par Diane Potvin, Montréal, Éditions France-Québec / (Paris), Fernand Nathan, (1979), 109 p. (coll. "Classiques du monde"l.

Rédition, avec notes infrapaginales, du texte de 1965.

37. Le Ciel de Québec, roman, (Montréal-Nord), VLB éditeur, (1979), 408 p.

Rédition du texte de'1969, accompagnée d'une "Table des principaux personnages historiques".

38. Gaspé-Mattempa, (Trois-Rivières), Éditions du Bien public, (1980), 52 p. (coll. "Choses et gens du pays").

Le récit, d'abord lu à Radio-Canada, le 18 août 1975, dans le cadre de la série "Un écrivain et son pays" (réseau MF), est précédé d'une lettre de Ferron à Clément Marchand. Une partie de l'oeuvre (p. 9-21) a été publié dans la revue SEM, en 1975.

39. Rosaire précédé de: I'Exécution de Maski, (Montréal-Nord), VLB éditeur, (1981) $197 \mathrm{p}$.

40. Cotnoir, conte (sic), (Montréal-Nord), VLB éditeur, (1981), $111 \mathrm{p}$.

Réédition du roman publié en 1962.

41. La Barbe de François Hertel, conte (sic), (Montréal-Nord), VLB éditeur, (1981), 54(2) p.

Réédition de la sotie publiée en 1951.

B. Pièces radiophoniques

1. "J'ai déserté Saint-Jean-de-Dieu".

Adaptation de la "Lettre d'amour" publiée en 1971. Diffusée au réseau MF de Radio-Canada, le 14 octobre 1971, dans le cadre de la série "Premieres"; réalisa tion de Madeleine Gérôme.

2. "Les Cartes de crédit".

Diffusée au réseau MF de Radio-Canada, le 14 novembre 1972, dans le cadre de la série "Premières"; réalisation de Madeleine Gérôme.

3. "Les Yeux".

Diffusée au réseau MF de Radio-Canada, le 4 octobre 1974, dans le cadre de la série "Premières"; réalisation d'André Major.

4. "La Ligue des bienfaiteurs de l'humanite".

Diffusée au réseau MF de Radio-Canada, le 28 février 1975, dans le cadre de la série "Premières"; réalisation de Madeleine Gérôme.

\section{Choix d'6tudes sur Jacques Ferron et son oeuvre}

\section{Essais et articles de périodiques.}

8OUCHER, Jean-Pierre, les "Contes" de Jacques Ferron, (Montréal), l'Aurore, (1974), 149 p. (coll. "I'Amélanchier: essai", 1). 
BOUCHER, Jean-Pierre, Jacques Ferron au pays des amélanchiers, Montréal, PUM, 1973, 112 p. (coll. "Lignés québécoises: textuelles").

CANTIN, Pierre, "Nouvelle contribution à la bibliographie des écrits de Jacques Ferron", dans Revue d'histoire littéraire du Québec et du Canada français, no 2 (1980-1981), Montréal, Ėditions Bellarmin, p. 115-135.

"Dossier Jacques Ferron", dans Québec français, no 15, (juin 1974). Repris dans Romanciers du Québec, (Québec), Éditions Québec français, 1980, p. 51-74.

"Jacques Ferron", numéro spécial de la revue Études françaises, vol. XII, no 3-4 loctobre 1976). (Articles de Jean-Marcel Paquette, Gilles Marcotte, Bernard Dupriez, Claude Filteau, Robert Mélançon, Donaid Smith, Robert Migner; bibliographie de Diane Potvin).

Jacques Ferron: dossier de presse 1950-1981, Sherbrooke, Bibliothèque du Séminaire de Sherbrooke, 1981, (s.p.).

L'HERAULT, Pierre, Jacques Ferron cartographe de l'imaginaire, Montréal, PUM, 1980, 293 p. (coll. "Lignes québécoises".)

MAlLHOT, Laurent, "Jacques Ferron: de l'amour incertain à la patrie possible", dans GODIN, Jean-Cléo et Laurent MAILHOT, Le Thétre québécois: introduction à dix dramaturges contemporains, (Montréal), Hurtubise HMH, (1970), p. 151-172.

MONETTE, Guy, Introduction à l'oeuvre de Jacques Ferron, (s. 1.), APFUC; (1982), 23 p. (coll. "Fascicules pédagogiques", 6).

(PAQUETTE), Jean Marcel, Jacques Ferron malgré lui, Montréal, Éditions du Jour, (1970), 221 p. (coll. "Littérature du Jour", Y-1); édition revue et augmentée, (Montréal), Ėditions Parti pris, (1978), 285 p. (coll. "Frères chasseurs", 1).

PAQUETTE, Jean-Marcel, "Jacques Ferron ou le Drame de la théattralite", dans le Théttre canadien-français: évolution, témoignages, bibliographie, Montréal, Fides, (1976), p. 581-596. (coll. "Archives des lettres canadiennes", 5).

ROUSSAN, Jacques de, Jacques ferron: quatre itinéraires, (Montréal), PUQ, (1971), 91 p. (coll. "Studio").

SMITH, Donald, "Jacques Ferron ou la folie d'écrire", dans les Lettres québécoises, no 6 (avril-mai 1977), p. 34-41.

TASCHEREAU, Yves, le Portuna: la médecine dans /"oeuvre de Jacques Ferron, (Montréall, l'Aurore, (1975), 120 p. (coll. "l'Amélanchier: essai", 3).

VANASSE, André, "le théátre de Jacques Ferron: à la recherche d'une identité", dans Livres et Auteurs québécois 1969, Québec, P.U.L., 1970 p. 219-230.

ZIROFF, Mary, A Study Guide to Jacques Ferron's "Tales from the Uncertain Country". (Toronto), Anansi, (1977), 25 p.

\section{Thdses et mémoires}

BEAUDOUX-NOIRCENT, Claudine. "Les transgressions progressives du conte dans la Charse du marechal ferrant", M.A., Sherbrooke, Université de Sherbrooke, 1980.

BEDNARSKI, Betty. "Le pays incertain de Jacques Ferron", M.A., Halifax, Dalhousie University, 1969. 
CANTIN, Pierre. "Jacques Ferron polygraphe: bibliographie descriptive et critique" Ph.D., Ottawa, Université d'Ottawa, 1981.

CLAVERIE, José. "Marat Sade, les Grands Soleils: étude comparative", M.A., Montréal, Université de Montréal, 1971.

CLOUTIER, André. "L'identité et l'évolution des personnage principaux dañs la Nuit et Papa Boss de Jacques Ferron", D.E.S., Québec, Université Laval, 1974.

COTE-LACHAPELLE, Aline. "Une stylistique narrative et discursive appliquée dans les Contes du pays.incertain de Jacques Ferron", M.A. Sherbrooke, Université de Sherbrooke, 1975.

GIROUX-LEUTENEGGER, Suzanne, "La notion de salut dans le Salut de I'Irlande", M.A., Montréal, McGill University, 1975.

JEAN, Julie. "Jacques Ferron, auteur satirique", M.A., Montréal, Université de Montréal, 1977.

LACROIX, Pierre. "Le théatre de Jacques Ferron: de l'illusion fondamentale au cocu fondamental", Kingston, Queen's University, 1971.

L'HERAULT, Pierre. "Le pays et l'imaginaire dans l'oeuvre de Jacques Ferron", Ph.D., Montréal, McGill University, 1977.

MICHAUD, Ginette. "L'arrière-texte: lecture de trois fictions autobiographiques de Jacques Ferron", M.A., Montréal, Université de Montréal, 1978.

MONETTE, Guy. "L'enclos maternel dans l'oeuvre romanesque de Jacques Ferron". M.A., Kingston, Queen's University, 1976.

MONETTE Guy. "L'oeuvre de Jacques Ferron et l'abandonnisme: essai de psychocritique", Ph.D. Kingston, Queen's University, 1981.

NADEAU, Dominique. "L'expérience de l'écriture dans la Charrette de Jacques Ferron", M.A., Montréal, McGill University, 1977.

NARDOCCHIO, Elaine F. “Les idéologies nationalistes de trois dramaturges québécois: Marcel Dubé, Jacques Ferron et Michel Tremblay", Ph.D., Québec, Université Laval, 1979.

PELLETIER, Noülle. "Le masque dans la Nuit de Jacques Ferron", M.A., Vancouver, University of British Columbia, 1976.

POTVIN, Diane. "Sémiologie de la variante chez Jacques Ferron: la Nuit et les Confitures de coings", M.A., Québec, Université Laval, 1980.

REILLY, Anne Thérèse. "Présence du conte dans Cotnoir et le Saint-Elias de Jacques Ferron". M.A., Vancouver, University of British Columbia, 1979.

SHOULDICE. Larry. "Contes anglais et autres de Jacques Ferron: introduction critique et traduction en anglais", M.A., Sherbrooke, Université de Sherbrooke, 1971.

SMITH, Donald. "Les idées sociales dans l'oeuvre de Jacques Ferron", Ph.D., Ottawa, Université d'Ottawa, 1979.

TASCHEREAU, Yves. "Le médecin et la médecine dans l'oeuvre de Jacques Ferron", M.A., Montréal, Université de Montréal, 1974.

TIBBLIN. Claudette. "Les Confitures de coings de Jacques Ferron: forme et significa tion", M.A.. Montréal, McGill University, 1975. 
VERDUYN, Christl. "L'oeuvre narrative de Jacques Ferron vue dans une perspective jungienne", M.A., Ottawa, Université d'Ottawa, 1976.

1. Diane Potvin, "Bibliographie des écrits de Jacques Ferron", dans Études françaises, vol. XII, no 3.4 loctobre 1976), p. (353)-383; reprise, avec quelques ajouts, dans l'ouvrage de Jean Marcel (Paquettel, Jacques ferron malgré lui, édition revue et corrigée, (Montréal), Parti pris, (1978), p. (253)-281. (coll. "Frères chasseurs", 11.

2. Pierre Cantin, "Nouvelle contribution à la bibliographie des ecrits de Jacques Ferron", dans la Revue d'histoire litteraire du Quebec et du Canada français, no 2 "Aspects et problemes", 1980-1981, Montréal, Éditions Bellarmin, p. (115)-135.

3. Intitulée "Jacques Ferron polygraphe: bibliographie descriptive et critique", notre thèse est présentement sous presse aux Éditions Bellarmin et devrait paraitre au début de 1983. Elle comprend une chronologie détaillée de la vie et de l'oeuvre de Jacques Ferron, ainsi que l'inventaire complet et mis à jour des écrits de l'auteur et de la critique jusqu'en mai 1982. 